\title{
Super-resolution imaging of alpha-synuclein polymorphisms and their potential role in neurodegeneration
}

\author{
Eileen Nugent ${ }^{1}$, Clemens F. Kaminski ${ }^{2}$, and Gabriele S. Kaminski Schierle ${ }^{2}$ \\ ${ }^{1}$ Sector of Biological and Soft Systems, The Cavendish Laboratory, University of Cambridge, \\ Cambridge CB3 OHE, United Kingdom ${ }^{2}$ Department of Chemical Engineering and \\ Biotechnology, University of Cambridge, Cambridge, Pembroke Street, Cambridge CB2 3RA, \\ United Kingdom
}

\begin{abstract}
:
The conversion of soluble, functional proteins into amyloid fibrils has been linked to the development of neurodegenerative disorders, including Parkinson's and Alzheimer's disease. In the brains of patients with these disorders, the increasing presence of amyloid-containing plaques corresponds to neuronal cell death and the worsening of symptoms. However, protein amyloids are not merely confined to dying cells. Rather, some show a propensity to be transmitted to, and enter adjacent cells and induce the polymerization of the native monomer population. Whether this process is directly associated with toxicity or not is still highly debated. In this mini review, we will discuss structural polymorphisms of $\alpha$-synuclein, as determined by super-resolution imaging techniques, and how these may be related to neuronal toxicity.
\end{abstract}

\section{Introduction:}

Alpha-synuclein is a small, intrinsically-disordered protein. It self-assembles into oligomers and subsequently into larger structures such as amyloid fibrils which proliferate in neurons during the progression of neurodegenerative disorders such as Parkinson's disease (PD). Recent experiments have demonstrated the propagation of native, wild-type $\alpha$-synuclein fibrils in the brains of mice demonstrating the capacity of normal proteins to contribute to the spread of PD pathology (1). In that work $\alpha$-synuclein fibrils of both human and mouse origin were injected into mouse brain cells resulting in a spreading, PD-like pathology. This "prion-like" ${ }^{1}$ propagation of $\alpha$-synuclein aggregates has led to a renewed interest in experimental techniques to investigate $\alpha$-synuclein polymorphisms.

In the prion field structural polymorphisms of prion proteins determine both their toxicity and infectivity (2) however the role of polymorphisms in amyloid related diseases is yet to be determined. To date, the role of polymorphism in toxicity for amyloid proteins (a class of proteins which include $\alpha$-synuclein) has only been shown for amyloid $\beta$ a protein related to Alzheimer's disease (6-8). Furthermore, repeated passage of amyloid through the same species of animal, an indication of infectivity, has so far not been observed for amyloid disease in contrast to prion diseases $(2,3)$ and increased expression of normal prion proteins does not cause a corresponding rise in disease pathology (2), whereas in PD increased $\alpha$-synuclein expression does (5). Much work remains to be done both on detecting amyloid polymorphisms, characterizing the heterogeneity of polymorphisms within cells and elucidating the role of polymorphisms in neurodegeneration.

\footnotetext{
${ }^{1}$ Prions are infectious agents made from protease-resistant misfolded isoforms of host protein.
} 
In prion diseases, where potential role of polymorphisms in neurodegenerative disorders was first uncovered, the need for high resolution imaging at the single molecule level to characterize heterogeneity has long been recognized. A seminal study was performed by DePace and Weissman in which the elongation rates of individual Sup35 fibrils were correlated with the presence of several distinct prion strains ${ }^{2}$ using atomic force microscopy (AFM)(9). Similar studies are lacking in the amyloid field where to date the majority of amyloid elongation kinetics experiments have been performed in vitro and measure the kinetics at the bulk rather than the single fibril level (10). In this mini-review we discuss new optical imaging techniques that have emerged to study the polymorphism of amyloids with single molecule resolution. We also summarise their application in studies both in vitro and in vivo and how their use has improved the understanding of amyloid pathology.

\section{Amyloid polymorphism, a definition:}

The production of functional proteins post translation is critically dependent on protein folding. Folding is sensitive to both, the primary structure of the protein and its cellular environment, and the probability of misfolding increases with mutations in the former or disruptions of the latter. Misfolded proteins are structurally distinct from their optimally folded counterparts and this distinction often implies a change in functionality. For example, functionality may decrease when misfolding is accompanied by a change in propensity of the protein to polymerise (or aggregate) leading to the range of possible macromolecular structures of the protein (polymorphism) some of which may be toxic.

Previous studies have shown that proteins such as Amyloid $\beta(A \beta)$ peptides can self-assemble into polymorphic fibrillary structures. In particular, in vitro, the morphology of the final fibrils and the extent of their heterogeneity (in terms of diameter, length, and degree of twisting) depend upon other factors including the absence or presence of agitation, $(11,12)$ protein concentration, $(13,14)$ physical properties, $(\mathrm{pH}(15)$ temperature $(16))$ and the composition of incubation medium $(15,17)$.

A subtler feature of the polymerisation process is the influence that can be exerted by preformed seed polymers, which are thought to act as structural templates for subsequent polymerisation and hence can influence polymorphism. It has indeed been shown that different types of seeding species leads to polymorphism $(11,12,18-20)$. In Jeong et al the authors claim that a mixture of $A \beta$ protofibrils and monomers leads to distinct, thicker fibrils that do not display properties found in non-templated fibrils such as secondary nucleation. Furthermore, in Pinotsi et al the authors show that a large subpopulation of pre-formed fibrils does not seed at all.

\section{Super-resolution imaging for the study of amyloid polymorphism:}

Optical super-resolution microscopy techniques are a powerful tool to study amyloid-related diseases: Using the technique of direct Stochastic Optical Reconstruction Microscopy, dSTORM (21), we were able to image in vitro and in cells the morphology of $A \beta$ fibrils with a resolution better than $20 \mathrm{~nm}$ (22). The technique thus affords a spatial resolution approaching that of AFM or SEM

\footnotetext{
${ }^{2}$ In the prion field "strain" refers to misfolded protein isolates that when transferred to another host cause prion disease pathology, the presence of several distinct prion strains would indicate polymorphism.
} 
techniques, whilst offering the advantages of specificity and sensitivity that come with all-optical techniques. In Figure 1 we show how dSTORM permits the tracking of fibril growth the single molecule level, offering completely new information on the self-assembly process, which is not accessible from bulk measurements (19).
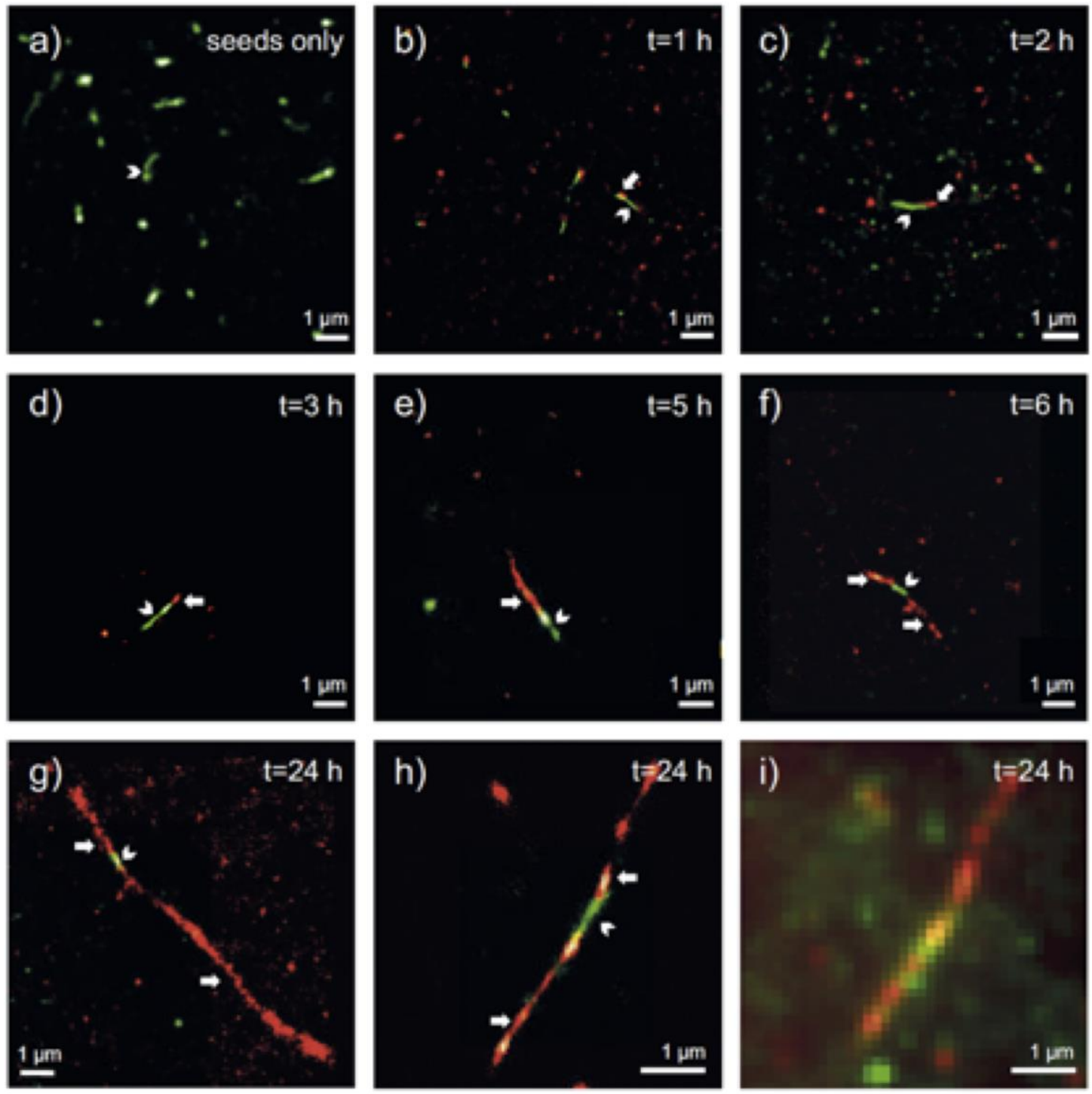

Figure 1: $\alpha$-synuclein elongation assay performed with $d$ STORM super-resolution microscopy. Small fibril seed species, shown in green, are incubated in solution containing monomeric $\alpha$-synuclein, shown in red. $\alpha$-synuclein seeds were covalently labelled with Alexa Fluor 568 and monomer with Alexa Fluor 647 dyes, respectively. The images show the time-sequenced growth of individual $\alpha-$ synuclein fibrils. Clearly, growth takes place from both ends of the seed fibril, extending to several micrometers in length after 24 hours. The last image shows a conventional fluorescence microscopy image, blurred by optical diffraction. Adapted with permission from (19). Copyright 2014 American Chemical Society.

We used small fibrillar seed species of $\alpha$-synuclein labelled with the Alexa Fluor ${ }^{\circledR} 568$ dye and incubated them in a solution of monomeric $\alpha$-synuclein that was labelled with Alexa Fluor ${ }^{\circledR} 647$ and 
studied fibril growth using dSTORM. Clearly, growth is seen for individual molecular species. Several new insights were obtained from this study that could not have been verified otherwise: First, fibril growth clearly proceeds from both ends of the fibril seeds, a hypothesis, that had been the subject of considerable scientific debate for lack of conclusive evidence. Secondly, we were able to show that the growth rates of individual fibrils varied by orders of magnitude; whilst some fibrils grew hardly at all, others extended over disproportional length over the same aggregation period(19). The experiment dismissed both random diffusion or the so called 'stop and go' models of monomer addition, and instead pointed to the likely existence of several 'misfolding states', for which aggregation can proceed, but which differ in growth rate. It is possible then with such techniques to verify whether certain toxic species can 'force' healthy species to co-aggregate etc. The latter is a variant of the "prion-like" propagation model of amyloid spreading in disease.

One of the advantages of super-resolution microscopy is that it is equally amenable to study amyloid fibril elongation in cells. Using $d$ STORM we have recently shown that similarly to our in vitro results described above elongation is favoured over nucleation in cells(23). In particular, we externally added green-labelled $\alpha$-synuclein seeds and red-labelled monomeric protein to differentiated $\mathrm{SH}$ SY5Y cells and observed that seed elongation was dominant over self-nucleation of red-labelled monomeric protein. Similarly, this was the case when we added only green-labelled seeds and determined seed elongation by endogenous $\alpha$-synuclein, naturally present in SH-SY5Y and embryonic rat ventral mesencephalic neurons, using species specific red- labelled antibodies and dSTORM to determine seed elongation. Importantly, toxicity, measured by induction of apoptosis in ventral mesencephalic neurons, did not occur upon incubation of neurons with seed fibrils but upon addition of monomeric synuclein. In contrast, seed fibrils were protective of neurons incubated with monomeric $\alpha$-synuclein. Moreover, our results showed that exogenously added monomer leads to the induction of endogenous $\alpha$-synuclein aggregation without the two moieties coming into close proximity. It thus remains to be determined whether it is cell stress induced by the addition of excess monomeric protein or small aggregates (which cannot be resolved by super-resolution imaging) that are causative of apoptosis. 

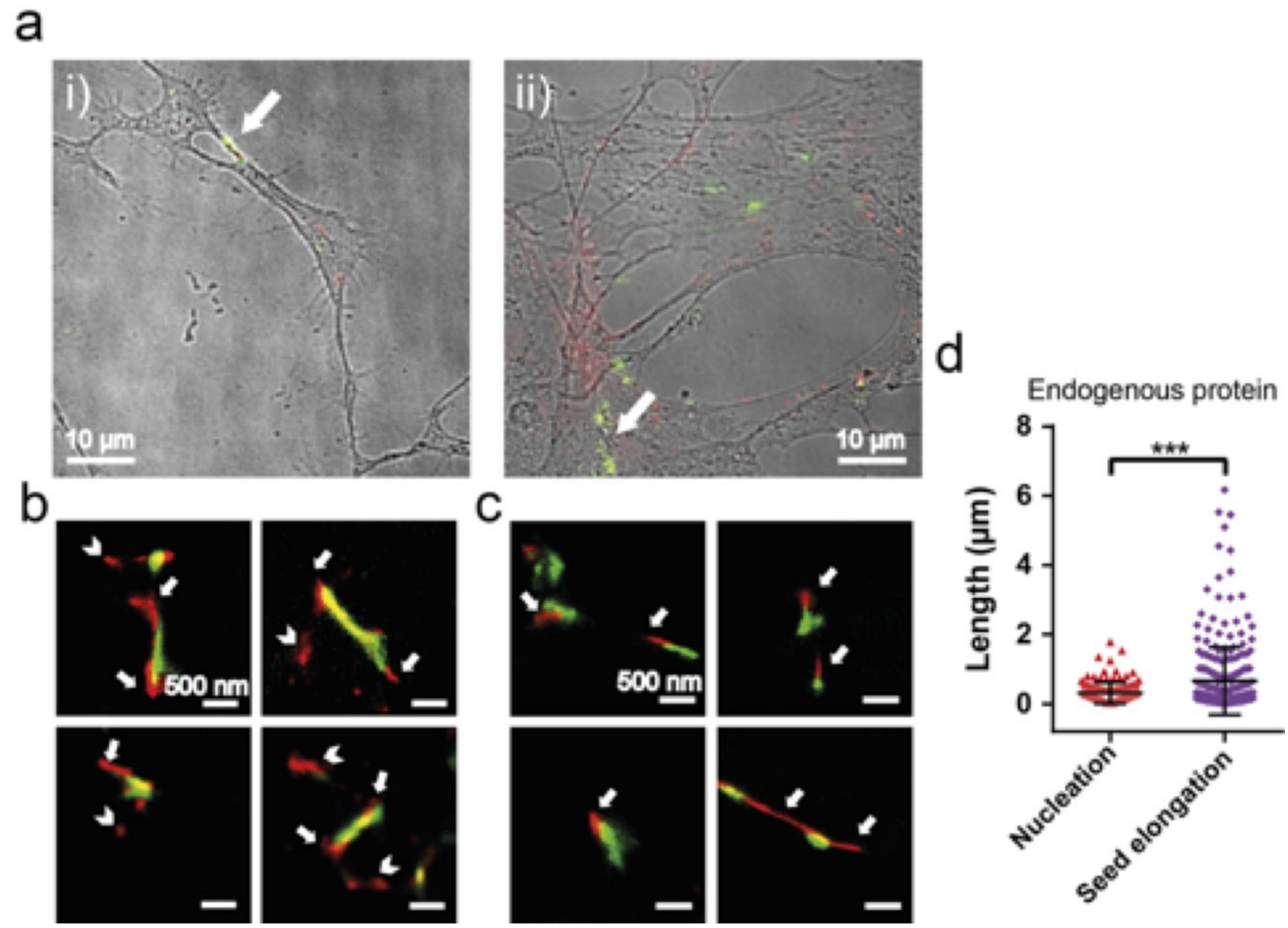

Figure 2: a) i) and ii) Overlaid DIC and wide-field fluorescence images of ventral-mesencephalic (VM) cells treated for $1 \mathrm{~h}$ with Alexa Fluor ${ }^{\circledast}$ 568-labeled $\alpha$-synuclein seed fibrils (green), incubated for $24 \mathrm{~h}$ in $\alpha$-synuclein -free medium and immunostained for endogenous $\alpha$-synuclein with a secondary antibody tagged with AF647 (red). b) Zoomed-in dSTORM images of hetero-fibrils formed of exogenous seeds (green) elongated by endogenous $\alpha$-synuclein (red, indicated by an arrow) in ventral mesencephalic cells. The top two images correspond to fibrils located in the areas indicated by the white arrows in (a). Scale bars correspond to $500 \mathrm{~nm}$. c) Zoomed-in dSTORM images of hetero-fibrils formed of exogenous seeds (green) elongated by endogenous $\alpha$-synuclein (red, indicated by an arrow) in SH-SY5Y cells. d) Quantification of the extent of seed elongation by endogenous $\alpha$-synuclein, from data indicated by arrows in (b) and (c) ("Seed elongation") and of the size of aggregates consisting of endogenous $\alpha$-synuclein only, as indicated by an arrowhead in (b) ("Nucleation"). The statistical analysis was performed using an unpaired t-test. ${ }^{* * *}$ : $p<0.001$. The experiment was repeated 8 times in SH-SY5Y cells and 7 times in primary ventral mesencephalic cell cultures and for each experiment at least 8 randomly chosen areas on the glass coverslip were imaged.

Based on our results one may distinguish two different mechanisms of pathology propagation: the transport of pre-formed aggregates, such as exogenously added seeds and an intracellular mechanism associated with fibril/aggregate formation which can occur upon increased levels of $\alpha$ synuclein in cells. The latter is highly toxic to the cell and while the former appears to be of relativity low toxicity the impact of this mechanism on cell survival on the timescale of years remains to be seen. Hints can be drawn from studies in patients who had received transplants of 'healthy' human 
embryonic ventral mesencephalic tissue to alleviate Parkinson-like symptoms $(24,25)$. In those studies, healthy brain tissue was placed in a host environment with a high proportional of pathological $\alpha$-synuclein aggregates. The timeframe for such healthy embryonic cells to have $\alpha$ synuclein inclusion pathology was over 10 years (26) and the patients displayed significant motor improvements without requiring pharmacological dopaminergic therapies up to 18 years after transplantation (27-29). This observation of two different mechanisms being involved in PD is similar observations in the prion field (2).

In summary, super-resolution microscopy has opened up the avenue for the study $\alpha$-synuclein polymorphism in vitro, a method that is extendable for similar studies inside a cellular environment. The method has further provided insight into fibril kinetics and could, for the first time, correlate the type of species with toxicity, highlighting that similar to prion-like diseases, there is a non-toxic mechanism of propagation versus one that causes toxicity within.

ACKNOWLEDGMENTS. This work was funded by grants from the UK Medical Research Council (MR/K015850/1 and MR/K02292X/1), Alzheimer's Research UK (ARUK-EG2012A-1), the UK Engineering and Physical Sciences Research Council (EP/H018301/1), and the Wellcome Trust (089703/Z/09/Z).

1. M. Masuda-Suzukake et al., Prion-like spreading of pathological $\alpha$-synuclein in brain. Brain. 136, 1128-38 (2013).

2. M. Halliday, H. Radford, G. R. Mallucci, Prions: generation and spread versus neurotoxicity. J. Biol. Chem. 289, 19862-8 (2014).

3. S. B. Prusiner et al., Evidence for $\alpha$-synuclein prions causing multiple system atrophy in humans with parkinsonism. Proc. Natl. Acad. Sci. U. S. A. 112, E5308-17 (2015).

4. F. Morinet, Prions: A model of conformational disease? Pathol. Biol. 62, 96-99 (2014).

5. M. J. Devine, K. Gwinn, A. Singleton, J. Hardy, Parkinson's disease and $\alpha$-synuclein expression. Mov. Disord. 26, 2160-8 (2011).

6. H. Vignaud et al., A Structure-Toxicity Study of Aß42 Reveals a New Anti-Parallel Aggregation Pathway. PLoS One. 8, e80262 (2013).

7. B. Winner et al., In vivo demonstration that -synuclein oligomers are toxic. Proc. Natl. Acad. Sci. 108, 4194-4199 (2011).

8. M. Stefani, Structural polymorphism of amyloid oligomers and fibrils underlies different fibrillization pathways: immunogenicity and cytotoxicity. Curr. Protein Pept. Sci. 11, 343-54 (2010).

9. A. H. DePace, J. S. Weissman, Origins and kinetic consequences of diversity in Sup35 yeast prion fibers. Nat. Struct. Biol. 9, 389-396 (2002).

10. M. S. Z. Kellermayer, A. Karsai, M. Benke, K. Soós, B. Penke, Stepwise dynamics of epitaxially growing single amyloid fibrils. Proc. Natl. Acad. Sci. U. S. A. 105, 141-144 (2008).

11. A. T. Petkova et al., Self-propagating, molecular-level polymorphism in Alzheimer's betaamyloid fibrils. Science. 307, 262-5 (2005).

12. A. K. Paravastu, I. Qahwash, R. D. Leapman, S. C. Meredith, R. Tycko, Seeded growth of betaamyloid fibrils from Alzheimer's brain-derived fibrils produces a distinct fibril structure. Proc. Natl. Acad. Sci. U. S. A. 106, 7443-8 (2009).

13. H. H. Bauer et al., Architecture and polymorphism of fibrillar supramolecular assemblies 
produced by in vitro aggregation of human calcitonin. J. Struct. Biol. 115, 1-15.

14. C. B. Andersen, D. Otzen, G. Christiansen, C. Rischel, Glucagon amyloid-like fibril morphology is selected via morphology-dependent growth inhibition. Biochemistry. 46, 7314-24 (2007).

15. H. Yagi, T. Ban, K. Morigaki, H. Naiki, Y. Goto, Visualization and classification of amyloid beta supramolecular assemblies. Biochemistry. 46, 15009-17 (2007).

16. R. Kodali, A. D. Williams, S. Chemuru, R. Wetzel, Abeta(1-40) forms five distinct amyloid structures whose beta-sheet contents and fibril stabilities are correlated. J. Mol. Biol. 401, 503-17 (2010).

17. C. Goldsbury, P. Frey, V. Olivieri, U. Aebi, S. A. Müller, Multiple assembly pathways underlie amyloid-beta fibril polymorphisms. J. Mol. Biol. 352, 282-98 (2005).

18. H. Komatsu, E. Feingold-Link, K. A. Sharp, T. Rastogi, P. H. Axelsen, Intrinsic linear heterogeneity of amyloid $\beta$ protein fibrils revealed by higher resolution mass-per-length determinations. J. Biol. Chem. 285, 41843-51 (2010).

19. D. Pinotsi et al., Direct observation of heterogeneous amyloid fibril growth kinetics via twocolor super-resolution microscopy. Nano Lett. 14, 339-45 (2014).

20. J. S. Jeong, A. Ansaloni, R. Mezzenga, H. A. Lashuel, G. Dietler, Novel mechanistic insight into the molecular basis of amyloid polymorphism and secondary nucleation during amyloid formation. J. Mol. Biol. 425, 1765-81 (2013).

21. M. Heilemann et al., Subdiffraction-resolution fluorescence imaging with conventional fluorescent probes. Angew. Chem. Int. Ed. Engl. 47, 6172-6 (2008).

22. G. S. Kaminski Schierle et al., In situ measurements of the formation and morphology of intracellular $\beta$-amyloid fibrils by super-resolution fluorescence imaging. J. Am. Chem. Soc. 133, 12902-5 (2011).

23. D. Pinotsi et al., Nanoscopic insights into seeding mechanisms and toxicity of $\alpha$-synuclein species in neurons. Proc. Natl. Acad. Sci. U. S. A. (2016), doi:10.1073/pnas.1516546113.

24. J.-Y. Li et al., Lewy bodies in grafted neurons in subjects with Parkinson's disease suggest host-to-graft disease propagation. Nat. Med. 14, 501-3 (2008).

25. J. H. Kordower, Y. Chu, R. A. Hauser, T. B. Freeman, C. W. Olanow, Lewy body-like pathology in long-term embryonic nigral transplants in Parkinson's disease. Nat. Med. 14, 504-6 (2008).

26. P. Brundin, J. H. Kordower, Neuropathology in transplants in Parkinson's disease: implications for disease pathogenesis and the future of cell therapy. Prog. Brain Res. 200, 221-41 (2012).

27. Z. Kefalopoulou et al., Long-term clinical outcome of fetal cell transplantation for Parkinson disease: two case reports. JAMA Neurol. 71, 83-7 (2014).

28. P. J. Hallett et al., Long-term health of dopaminergic neuron transplants in Parkinson's disease patients. Cell Rep. 7, 1755-61 (2014).

29. S. Grealish et al., Human ESC-Derived Dopamine Neurons Show Similar Preclinical Efficacy and Potency to Fetal Neurons when Grafted in a Rat Model of Parkinson's Disease. Cell Stem Cell. 15, 653-665 (2014). 